\title{
Association between Oral Microbiome and Esophageal Diseases: A State-of-the-Art Review
}

\author{
Rachel Bernard $^{a}$ Irtiqa Fazili ${ }^{b}$ Seesandra V. Rajagopalac Suman R. Das ${ }^{d}$ \\ Girish Hiremath $^{\mathrm{a}}$ \\ aDivision of Pediatric Gastroenterology, Hepatology, and Nutrition, Monroe Carrell Jr Vanderbilt Children's Hospital, \\ Nashville, TN, USA; ' University of Tennessee Health Science Center, Memphis, TN, USA; 'Division of Infectious Diseases, \\ Department of Medicine, Vanderbilt University Medical Center, Nashville, TN, USA; ${ }^{\mathrm{d}}$ Department of Otolaryngology and \\ Department of Pathology Microbiology and Immunology, Vanderbilt University Medical Center, Nashville, TN, USA
}

\section{Keywords \\ Human microbiome · Esophageal diseases · Esophageal neoplasms · Gastrointestinal microbiome · Barrett's esophagus}

\begin{abstract}
Background: Esophageal conditions result in significant morbidity and mortality worldwide. There is growing enthusiasm for discerning the role of microbiome in esophageal diseases. Conceivably, the focus has been on examining the role of local microbiome in esophageal diseases although this is somewhat limited by the invasive approach required to sample the esophageal tissue. Given the ease of sampling the oral cavity combined with the advances in genomic techniques, there is immense interest in discovering the role of the oral microbiome in esophageal conditions. Summary: In this review, we aim to discuss the current evidence highlighting the association between the oral microbiome and esophageal diseases. In particular, we have focused on summarizing the alterations in oral microbiome associated with malignant, premalignant, and benign esophageal cancers, inflammatory and infectious conditions, and esophageal dysmotility diseases. Identifying alterations in the oral microbi-
\end{abstract}

ome is a key to advancing our understanding of the etiopathogenesis and progression of esophageal diseases, promoting novel diagnostics, and laying the foundation for personalized treatment approaches. Key Messages: Further studies are needed to unravel the mechanisms by which the oral microbiome influences the development and progression of esophageal diseases, as well as to investigate whether alterations in the oral microbiome can impact the natural history of various esophageal diseases.

(c) 2021 S. Karger AG, Basel

\section{Introduction}

In the US alone, it is estimated that 18,440 new cases of esophageal cancer will be diagnosed in 2020 and 16,170 deaths are expected from the disease [1]. Likewise, the incidence, prevalence, and the health-care burden related to the benign esophageal disorders such as eosinophilic esophagitis (EoE) and gastroesophageal reflux disease (GERD) have significantly increased within the past 2 decades, particularly in the western hemisphere $[2,3]$. At present, histologic examination of esophageal samples obtained via endoscopy remains the mainstay of diagnos- karger@karger.com

www.karger.com/ddi (c) 2021 S. Karger AG, Basel
Correspondence to:

Girish Hiremath, girish.hiremath@vumc.org$$
\text { 监 }
$$ \\ Karger"}


ing esophageal pathology. However, this approach is invasive, burdensome, and expensive. As such, there is a crucial need to identify and develop newer and efficient approaches to advance diagnostics as well as to improve our understanding of the pathobiology of esophageal diseases to ultimately improve clinical outcomes.

While pathogenesis of most of the esophageal diseases remains to be fully delineated, the etiological studies have focused on the interaction between hereditary probability, environmental factors, the host immune regulation, and the commensal microbiome. In the recent years, there has been growing interest in uncovering the role of the microbiome in esophageal diseases. These efforts have been accelerated by the advances in genomic techniques. These technological developments have allowed us to simultaneously explore numerous pieces of the microbial microenvironment including microbial make up and function as well as to infer community function [4]. For instance, the use of next-generation sequencing technology has helped to illuminate the genetic variations and the interconnection between the host and pathogen $[5,6]$.

The local dysbiosis has been well studied in esophageal disorders $[7,8]$. Emerging evidence suggests that the inherent esophageal microbiome is comparable to the oral microbiome but with key taxonomic differences [9-11]. While the association between oral microbiome and oral, oropharyngeal, and gastrointestinal cancers has been previously published, the association between alterations in the oral microbiome and esophageal diseases has not been reviewed [12-14].

The human oral microbiota, defined as all the microorganisms that are found on or in the human oral cavity and its contiguous extensions (stopping at the distal esophagus), have been well studied [15]. The oral cavity has over 775 bacterial species [16] and its composition is suspected to be affected by many variables including host genetics [17], geography [18], age [19], oral health [20], lifestyle habits [21], social factors [22], and medications [23]. Studies investigating the composition of the oral microbiota in healthy individuals have demonstrated a predominance of gram-positive organisms including Abiotrophia, Peptostreptococcus, Streptococcus, Stomatococcus, Actinomyces, Bifidobacterium, Corynebacterium, Eubacterium, Lactobacillus, Propionibacterium, Pseudoramibacter, and Rothia [16]. Gram-negative organisms colonized in the healthy oral microbiome include Moraxella, Neisseria, Veillonella, Campylobacter, Capnocytophaga, Desulfobacter, Desulfovibrio, Eikenella, Fusobacterium, Hemophilus, Leptotrichia, Prevotella, Selemonas, Simonsiella, Treponema, and Wolinella [16]. In this narrative review, we aimed to summarize the literature describing the associations between alterations in the oral microbiome and esophageal diseases in humans.

\section{Methods}

\section{Search Strategy}

To identify relevant studies, we searched publications in PubMed, Google Scholar, and Web of Science corresponding to the "PEO" format (population = human, exposure = oral microbiome, outcome = esophageal diseases) which is recommended for a narrative review [24]. In order to optimize the search for relevant publications, no date limits were imposed. A combination of search terms such as oral microbiome (or microbiota), human oral microbiome (or microbiota), salivary microbiome (or microbiota), oral bacteria, oral bacterial diversity, microbiome (or microbiota) of the oropharynx, oral swabs, and esophageal (or esophageal) diseases, esophageal (or esophageal) malignancies or cancers (including esophageal adenocarcinoma [EAC], esophageal squamous cell carcinoma [ESCC]), Barrett's esophagus (BE), EoE, GERD, reflux esophagitis (or esophagitis), esophageal (or esophageal) infections (due to candidiasis, herpes virus, and cytomegalovirus), esophageal (or esophageal) achalasia, and megaesophagus (or megaesophagus) were used.

\section{Inclusion and Exclusion Criteria}

We included publications which reported changes in the oral microbiome in adult and pediatric patients with the esophageal diseases listed above. To ensure quality, nonoriginal articles, nonhuman studies, and abstract-only publications were excluded. Additionally, studies that focused on the esophageal microbiome alone compared the esophageal microbiome with changes in the microbiome at other sites other than the oral microbiome, or those published in languages other than English were also excluded.

\section{Data Extraction}

Two authors (I.F. and R.B.) evaluated articles for eligibility and quality and abstracted data independently according to the guidelines [25]. A standardized data extraction form was designed to collect: title, author, publication year, country of origin, study type, specific aims, research methods, and conclusions. Statistical outcomes related to the average species diversity (alpha diversity, Chaol or ACE index, and Mantel test), the extent of change in community composition (beta diversity, Shannon diversity index, and Simpson diversity index), percentage of relative abundance, number of operational taxonomic units (OTUs), and area under the receiver operating characteristic were extracted when available. Any disagreement between authors in data abstraction was resolved by discussion with the senior author (G.H.) and review of the publication(s). This approach allowed for minimization of the risk of bias.

\section{Results}

\section{Study Selection}

Forty-six publications were identified. Of these, 28 publications were discarded after reviewing their title and 
abstracts and 18 full articles were assessed for eligibility. Of these, 16 articles were included in the narrative review and qualitative synthesis (Fig. 1). Our major findings are summarized in Table 1 and Figure 2.

\section{Oral Microbiome in Esophageal Malignancies}

Narikiyo et al. [26] for the first time reported specific oral bacteria associated with esophageal malignancy. By cloning the $16 \mathrm{~S}$ rRNA gene amplicons into a shuttle vector and then sequencing the $16 \mathrm{~S}$ amplicon clones to identify representative bacterial taxon, they analyzed saliva collected from Japanese subjects with uncategorized esophageal carcinoma and healthy controls. They found that Treponema denticola (45\%) and Streptococcus anginosus (12\%) were abundant in saliva collected from esophageal cancer patients and were absent in the healthy controls. Subsequently, they analyzed esophageal cancer tissue samples collected from subjects living in other countries and found similar results suggesting the abundance of salivary T. denticola and S. anginosus in subjects with esophageal cancer was not unique to Japanese patients. Recently, Kageyama et al. [27] conducted a casecontrol study examining the salivary microbiota in patients with different gastrointestinal tract cancers, including 12 with unspecified esophageal cancer and their age- and sex-matched controls. The OTUs corresponding to Porphyromonas gingivalis (estimated relative abundance: $0.3 \%$ vs. $0.0 \%, p<0.01$ ), Corynebacterium species $(0.5 \%$ vs. $0.1 \%$ relative abundance, $p<0.01)$, and Fusobacterium nucleatum subspecies vincentii ( $0.45 \%$ vs. $0.1 \%, p<0.05)$ were more abundant in the saliva of esophageal cancer patients compared to that of the healthy controls. Additionally, $16 \mathrm{~S}$ rRNA gene sequencing of the saliva samples demonstrated a significantly higher salivary bacterial diversity in esophageal cancer patients than healthy controls (number of OTUs, $p=$ 0.02 ; Shannon Index, $p<0.01$; Chao $1, p=0.04)$. Zhao et al. [28] also recently evaluated alterations of the oral microbiota in 39 uncategorized esophageal cancer patients and 51 healthy volunteers via mouth rinses. $16 \mathrm{~S}$ rRNA sequencing revealed the differential abundance of several species between the 2 groups. The esophageal cancer group demonstrated increased Firmicutes, Negatividcutes, Selenmonadales, Prevotellaceae, Prevotella, and Veillonellaceae taxa. Conversely, Protecobacteria, Betaproteobacerita, Neisseriales, Neisseriaceae, and Neisseria taxa were decreased. However, there was no significant difference in alpha diversity between the esophageal cancer and healthy control group (Shannon, $p=0.2$; Simpson, $p=0.071)$.

Oral Microbiome and Esophageal Diseases

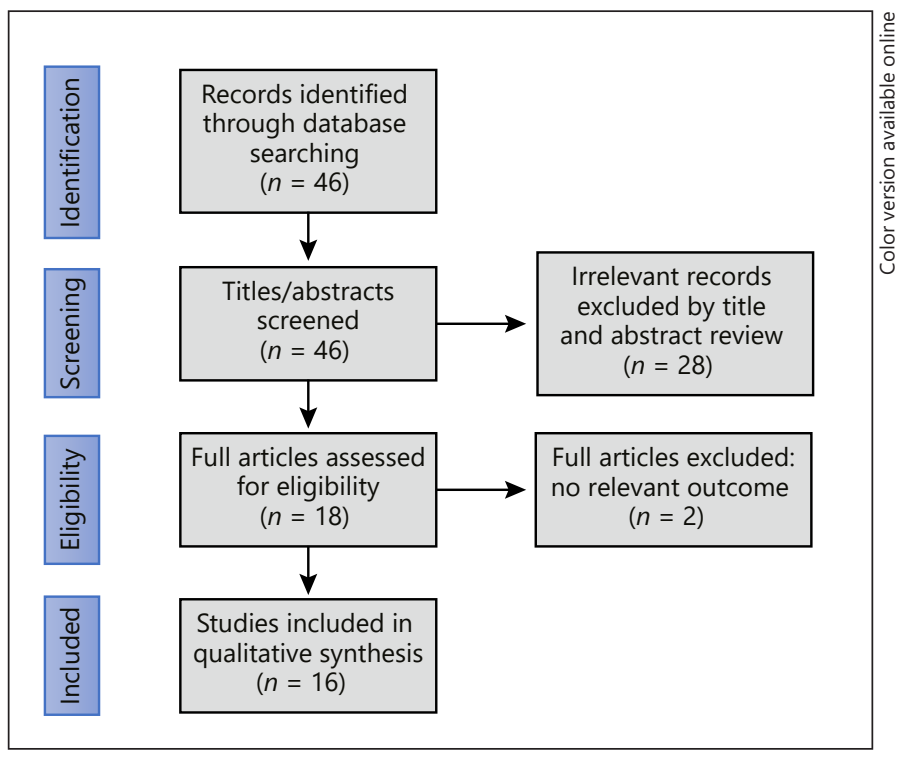

Fig. 1. Schematic illustration of the literature search.

In 2015, Chen et al. [29] focused on oral microbiome associations with ESCC. In this study, fasting saliva samples were collected from 87 incident and histopathologically confirmed ESCC patients, 63 subjects with dysplasia, and 85 healthy controls. Using $16 \mathrm{~S}$ rRNA gene sequencing, they observed a higher relative abundance of Streptococcus (percent abundance $21.9 \%$ vs. $16.1 \%, p<$ $0.01)$, Prevotella ( $42.4 \%$ vs. $36.1 \%, p<0.01$ ), and Porphyromonas $(8.9 \%$ vs. $6.5 \%, p<0.01)$ in the ESCC patients when compared to the non-ESCC patients (including subjects with dysplasia and healthy controls). The ESCC patients also had decreased carriage of Lautropia, Bulleidia, Cantonella, Corynebacterium, Moryella, Peptococcus, and Cardiobacterium. Furthermore, ESCC patients had an overall lower or comparable relative abundance of most of the other genera and decreased microbial diversity compared to those with esophageal dysplasia or healthy controls $(p<0.001)$. Likewise, Wang et al. [30] also utilized 16S rRNA sequencing to investigate saliva samples collected from 20 patients with ESCC and 21 healthy controls. They found that the ESCC patients had a higher proportion of Firmicutes (estimated relative abundance: $0.50 \%$ vs. $0.40 \%)$, Bacillus ( $0.30 \%$ vs. $0.20 \%$ ), Lactobacillus ( $0.25 \%$ vs. $0.20 \%)$, and a decreased abundance of Gammaproteobacteria ( $0.25 \%$ vs. $0.30 \%$ ) compared to the healthy controls. The diversity and richness in the ESCC samples trended to be lower than those of the healthy control group but these differences did not achieve statistical significance (Ace, Chao1, Shannon in- 
Table 1. Summary of studies applying oral microbiome analysis in esophageal diseases

\begin{tabular}{llll}
\hline Condition & Author & Methods & Outcomes \\
\hline $\begin{array}{l}\text { Esophageal } \\
\text { carcinoma }\end{array}$ & Narikiyo et al. [26] & $\begin{array}{l}\text { Sample size: not provided } \\
\text { Saliva samples from patients with esophageal carcinoma } \\
\text { and healthy controls }\end{array}$ & $\begin{array}{l}\text { Higher abundance: Treponema denticola and } \\
\text { 16S rRNA gene PCR amplicons were cloned into a shuttle } \\
\text { vector and then one hundred clones were sequenced }\end{array}$
\end{tabular}

\begin{tabular}{|c|c|c|}
\hline $\begin{array}{l}\text { Esophageal } \\
\text { carcinoma }\end{array}$ & Kageyama et al. [27] & $\begin{array}{l}\text { Sample size: } n=24 \\
\text { Saliva samples from } 59 \text { DTC patients ( } n=12 \text { with } \\
\text { esophageal cancer) and } 118 \text { age-/sex-matched controls } \\
\text { 16S rRNA gene sequencing (V1 to V2 region) }\end{array}$ \\
\hline $\begin{array}{l}\text { Esophageal } \\
\text { carcinoma }\end{array}$ & Zhao et al. [28] & $\begin{array}{l}\text { Sample size: } n=90 \\
\text { Mouth rinses from } 39 \text { esophageal carcinoma patients and } \\
51 \text { healthy controls } \\
\text { 16S rRNA gene sequencing (V3 to V4 region) }\end{array}$ \\
\hline
\end{tabular}

Higher abundance: Porphyromonas gingivalis

Corynebacterium species and Fusobacterium nucleatum subspecies vincentii

Diversity: increased

Higher abundance: Firmicutes, Negatividcutes,

Selenmonadales, Prevotellaceae, Prevotella, and Veillonellaceae taxa

Lower abundance: Protecobacteria, Betaproteobacerita

Neisseriales, Neisseriaceae, and Neisseria

Diversity: no significant difference

$\begin{array}{ll}\text { ESCC Chen et al. [29] } & \text { Sample size: } n=235 \\ & \text { Saliva samples from } 87 \text { ESCC cases, } 63 \text { dysplastic cases, }\end{array}$

Higher abundance: Streptococcus, Prevotella, and Porphyromonas

and 85 healthy controls

16S rRNA gene sequencing (V3 to V4 region)

Lower abundance: Lautropia, Bulleidia, Catonella,

Corynebacterium, Moryella, Peptococcus, and

Cardiobacterium

Diversity: decreased

\begin{tabular}{llll}
\hline ESCC Wang et al. [30] & Sample size: $n=41$ & $\begin{array}{l}\text { Higher abundance: Firmicutes, Bacillus, and Lactobacillus } \\
\text { Saliva samples from } 20 \text { ESCC and } 21 \text { healthy controls }\end{array}$ & $\begin{array}{l}\text { Lower abundance: Gammaproteobacteria } \\
\text { Diversity: no significant difference }\end{array}$ \\
& 16S rRNA gene sequencing (V3 to V4 region) & Higher abundance: Porphyromonas, Streptococcus, and \\
\hline ESCC & Meng et al. [32] & $\begin{array}{l}\text { Sample size: } n=52 \\
\text { Saliva samples from } 30 \text { ESCC and } 22 \text { healthy controls }\end{array}$ & Leptotrichia
\end{tabular}

$16 \mathrm{~S}$ rRNA gene sequencing (V4)

\begin{tabular}{llll}
\hline ESCC & Chen et al. [31] & $\begin{array}{l}\text { Sample size: } n=52 \\
\text { Oral biofilms from } 34 \text { ESCC and 18 healthy controls } \\
\text { 16S rRNA gene sequencing (V3-V4) }\end{array}$ & $\begin{array}{l}\text { Higher abundance: Streptococcus species, Veillonella } \\
\text { parvula, and P. gingivalis } \\
\text { Diversity: significant difference }\end{array}$ \\
\hline ESCC/EAC & Peters et al. [33] & $\begin{array}{l}\text { Sample size: } n=316 \\
\text { Mouthwash samples from } n=81 / 160 \text { EAC and } n=25 / 50\end{array}$ & $\begin{array}{l}\text { Porphyromonas gingivalis associated with a higher risk } \\
\text { of ESC }\end{array}$ \\
& & ESCC cases/matched controls & Tannerella forsythia associated with a higher risk of EAC \\
& & 16S rRNA gene sequencing (V4 region) & Depletion of Neisseria and Streptococcus pneumonia \\
& & associated with a lower risk of EAC & Diversity: no significant difference
\end{tabular}

\begin{tabular}{lll}
\hline ESCC/EAC Kawasaki et al. [34] & Sample size: $n=123$ & Higher abundance: A. actinomycetemcomitans and S. \\
& Saliva samples from 61 esophageal cancer (58 with ESSC anginosus &
\end{tabular}

and 3 with EAC) and 62 healthy controls

and 3 with EAC) and 62 healthy controls

Real-time PCR to calculate the density of $A$.

actinomycetemcomitans, Fusobacterium nucleatum, $P$.

gingivalis, Prevotella intermedia, T. denticola, T. forsythia, and

S. anginosus

BE Snider et al. [35] Sample size: $n=49$

Saliva samples from 32 BE patients and 17 control patients

16S rRNA sequencing gene (V4 region)

Higher abundance: Firmicutes

Lower abundance: Proteobacteria

The linear regression model with the greatest

discrimination between $B E$ and controls included

Lautropia, Streptococcus, and an unspecified genus of

the order Bacteroidales

Diversity: no significant difference

\begin{tabular}{ll}
\hline BE Okereke et al. [36] & Sample size: $n=17$ \\
& Swabs from uvula and endoscope plus endoscopic \\
& biopsies from proximal, middle, distal, and BE \\
& 16S rRNA gene sequencing (V1 to V8 regions)
\end{tabular}

Higher abundance: Fusobacterium, Prevotella, and

Dialister in the uvular swabs

Streptococcus was detected but in a lesser quantity 
Table 1 (continued)

\begin{tabular}{|c|c|c|c|}
\hline Condition & Author & Methods & Outcomes \\
\hline EoE & Benitez et al. [37] & $\begin{array}{l}\text { Sample size: } n=68 \\
\text { Oral swabs and esophageal biopsies from } 33 \text { EoE patients } \\
\text { and } 35 \text { controls } \\
\text { 16S rRNA gene sequencing (V1 to V2) }\end{array}$ & $\begin{array}{l}\text { Higher abundance: Streptococcus, Neisseria, and } \\
\text { Prevotella }\end{array}$ \\
\hline EoE & Hiremath et al. [39] & $\begin{array}{l}\text { Sample size: } n=35 \\
\text { Saliva samples from } 26 \text { children with EoE and } 19 \text { controls } \\
\text { 16S rRNA gene sequencing (V4 region) }\end{array}$ & $\begin{array}{l}\text { Higher abundance: Streptococcus was more abundant } \\
\text { with active EoE versus non-EoE controls, Haemophilus } \\
\text { was more abundant in active EoE versus inactive EoE } \\
\text { Diversity: no significant difference }\end{array}$ \\
\hline $\begin{array}{l}\text { GERD } \\
\text { and EoE }\end{array}$ & $\begin{array}{l}\text { Norder Grusell et al. } \\
\text { [40] }\end{array}$ & $\begin{array}{l}\text { Sample size: } n=27 \\
\text { Oral punch biopsies and brush samplings from the oral } \\
\text { cavity (as well as brush samplings and endoscopy } \\
\text { biopsies of upper and lower esophagus) of } 17 \text { patients } \\
\text { with GERD and } 10 \text { with EoE } \\
\text { Culture }\end{array}$ & $\begin{array}{l}\text { Higher abundance: Streptococcus (viridians) was the } \\
\text { most common bacteria in both groups } \\
\text { Diversity: decreased in GERD versus EoE }\end{array}$ \\
\hline GERD & Wang et al. [43] & $\begin{array}{l}\text { Sample size: } n=106 \\
\text { Saliva samples from } 55 \text { reflux esophagitis and } 51 \text { controls } \\
\text { 16S rDNA gene sequencing (V3-V4 region) }\end{array}$ & $\begin{array}{l}\text { Higher abundance: Prevotella, Veillonella, Megasphaera, } \\
\text { Peptostreptococcus, Atopobium, Oribacterium, } \\
\text { Eubacterium, and Lachnoanaerobaculum } \\
\text { Lower abundance: Neisseria, Streptococcus, Rothia, } \\
\text { Granulicatella, Gemella, Aggregatibacter, Treponema, } \\
\text { Campylobacter, Filifactor, Corynebacterium, and Lactivibrio } \\
\text { Diversity: no significant difference }\end{array}$ \\
\hline
\end{tabular}

ESCC, esophageal squamous cell carcinoma; EAC, esophageal adenocarcinoma; DTC, digestive tract cancers; BE, Barrett's esophagus; EoE, eosinophilic esophagitis; GERD, gastroesophageal reflux disease.

dex, Simpson index all $p>0.05)$. In addition, Chen et al. [31] recently used 16S rRNA sequencing to investigate the esophageal microbiome from oral biofilms obtained from 34 patients with ESCC and 18 healthy donors by swabbing the dental plaque at the gingival margin on the molars with sterilized toothpicks. Results showed the alpha and beta diversity differed amongst ESCC and healthy donors. Specifically, Streptococcus species, Veillonella parvula, and P. gingivalis were more abundant in the oral biofilms of ESCC patients than in those of healthy volunteers. Similarly, Meng et al. [32] evaluated saliva samples from 30 ESCC patients together with 22 healthy controls. $16 \mathrm{~S}$ rRNA sequencing results showed that Porphyromonas $(p=0.001)$, Streptococcus $(p=0.01)$, and Leptotrichia $(p=0.009)$ were the most abundantly enriched in the ESCC saliva.

In a case-control study, Peters et al. [33] took the investigation of the oral microbiome in esophageal cancers a step further by including ESCC and EAC patient. Using $16 \mathrm{~S}$ rRNA gene sequencing, they compared mouthwash samples from 25 ESCC patients to 50 matched controls, and 81 EAC patients to 160 matched controls. They found that the abundance of the periodontal pathogen Porphyromonas gingivalis trended with a higher risk of ESCC (odds ratio $95 \%$ confidence interval $[95 \% \mathrm{CI}]=1.30$ [0.96-1.77], $p=0.09$ ). Additionally, the abundance of the periodontal pathogen, Tannerella forsythia, was positively associated with the risk of EAC (relative risk [95\% CI]: 1.21 [1.01-1.46], $p=0.04$ ) and depletion of the commensal genus Neisseria, and the species Streptococcus pneumoniae were associated with a lower risk of EAC (all $p<$ 0.05 ). Alpha and beta diversity did not differ significantly from matched controls in the EAC or ESCC cases. Additionally, Kawasaki et al. [34] investigated subgingival dental plaques and unstimulated saliva from 61 patients with esophageal cancer ( 58 with ESCC and 3 with EAC) and 62 cancer free age-matched individuals. Using bacteria gDNA and real-time PCR to calculate the bacterial copy numbers for 6 preselected bacterial species, the saliva samples demonstrated an increased prevalence $(p<$ 


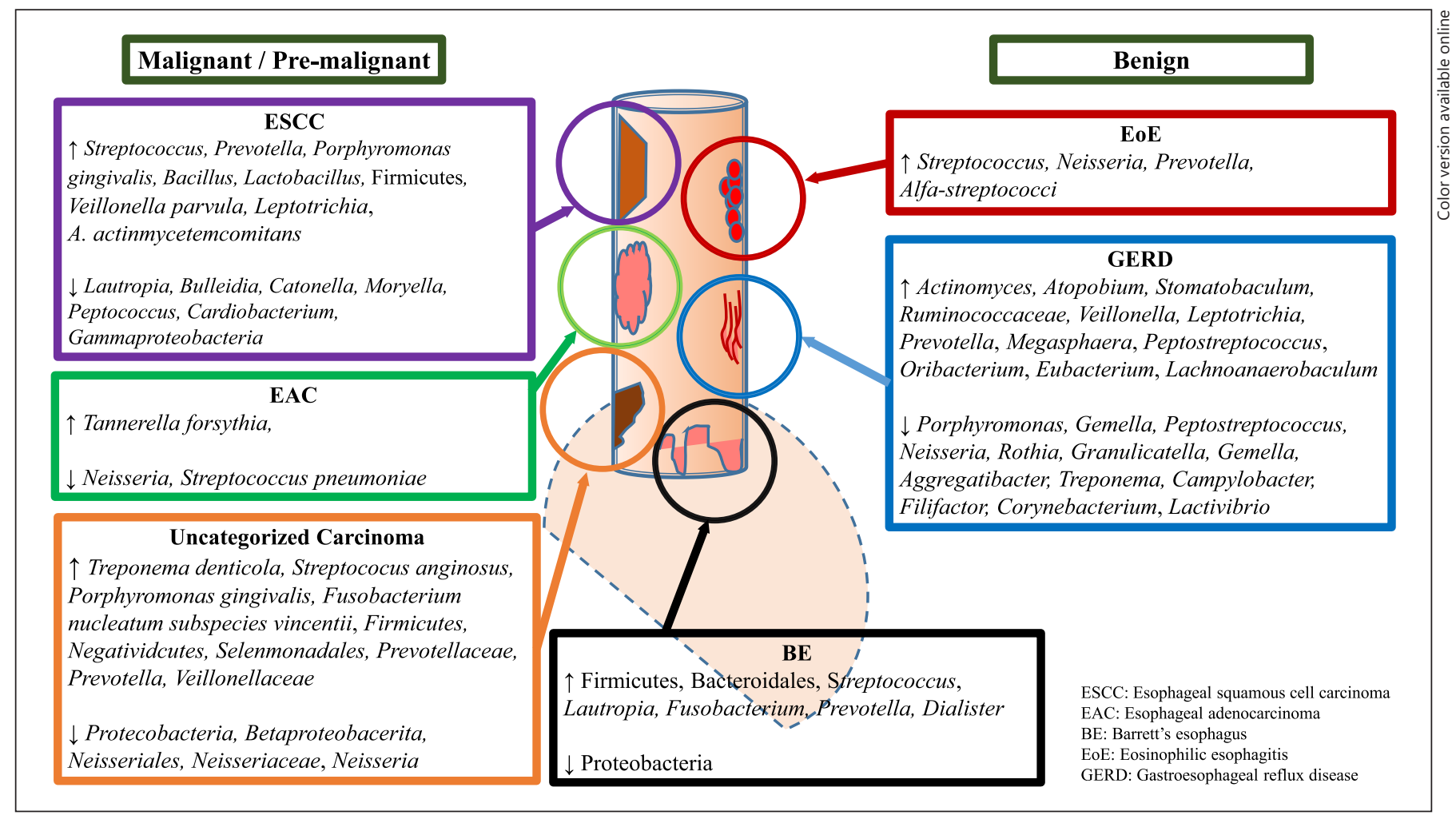

Fig. 2. Summary of changes in the oral microbiome in various esophageal diseases.

$10^{-3}$ ) and number of copies of Aggregatibacter actinomycetemcomitans in the esophageal cancer patients compared to the control patients $(p=0.001)$. S. anginosus was also significantly increased in the esophageal cancer patients $(p=0.004)$.

Oral Microbiome in Premalignant Esophageal Diseases

Two recent studies have examined the composition of the oral microbiome in BE. In one study, Snider et al. [35] collected saliva samples from a total of 49 patients prior to their endoscopy of whom 32 had BE and 17 were controls. 16S rRNA gene sequencing of these saliva samples revealed that at the phylum level, the oral microbiome in BE patients had high relative abundance of Firmicutes ( $27.1 \%$ vs. $14.6 \% ; p=0.005)$ and decreased abundance of Proteobacteria ( $23.8 \%$ vs. $34.5 \%$; $p=0.02)$. When comparing patients with $\mathrm{BE}$ to controls, the results demonstrated a relative abundance of Lautropia, Streptococcus, and a genus in the order Bacteroidales, which the authors suggest could be used to precisely identify BE with high sensitivity (96.9\%) and specificity (88.2\%) (area under the receiver operating characteristic of 0.94; 95\% CI: $0.085-$ $1.00 ; p=0.04$ vs. Lautropia alone). However, they did not observe any differences in the average species diversity (alpha diversity) when comparing patients with $\mathrm{BE}$ to controls (mean Shannon index: BE 2.73 vs. controls 2.89; $p=0.10$ ). In another study, Okereke et al. [36] obtained samples from the esophagus (distal, middle, proximal, and BE) as well as collected swabs from the uvula and the endoscope in 17 patients with BE. While the study compared the different techniques (i.e., uvula swab vs. endoscope swab vs. tissue biopsy) and their associated microbiome, the uvular swabs, which reflected the oral microbiome composition, showed a predominance of gram-negative organisms. In particular, $16 \mathrm{~S}$ rRNA sequencing demonstrated Fusobacterium (estimated 30\%), Prevotella (30\%), and Dialister (15\%) had the highest relative proportions in patients with BE. Interestingly, Streptococcus (5\%) was detected but in a lesser quantity.

\section{Benign Esophageal Diseases}

Benitez et al. [37] was the first group to study the oral and esophageal microbiota in EoE. In their study involving children, they used 16S rRNA gene sequencing to compare contents of the oral swabs and esophageal biopsies collected from 35 non-EoE controls and 33 EoE pa- 
tients. Of the 33 children with EoE, 18 had active EoE (defined as $\geq 15$ eosinophils per high power field per the 2011 consensus recommendations) [38] and 15 had inactive EoE (defined as $<15$ eosinophils per high power field). Although this study focused on the esophageal microbiota and its comparison to the oral microbiota, the study established that both oral and esophageal environments were predominantly composed of Streptococcus, Neisseria, and Prevotella (Mantel correlation $=0.16$, $p$ val ue: 0.008; Procrustes $R^{2}: 0.15, p$ value: 0.009). More recently, Hiremath et al. [39] evaluated saliva samples from 26 children with EoE and 19 non-EoE age- and ethnicitymatched controls. Their salivary microbiome was profiled using $16 \mathrm{~S}$ rRNA gene sequencing and was compared to validated EoE disease activity indices. This study found consistent evidence that Streptococcus trended to be abundant in the oral microbiome, more specifically in children with active EoE compared with non-EoE controls $(q$ value $=0.06)$. The relative abundance of Haemophilus was significantly higher in children with active EoE compared with inactive EoE ( $q$ value $=0.0008)$. Furthermore, when comparing the saliva samples from children with EoE to non-EoE controls, there was a trend toward lower alpha diversity and microbial richness in children with EoE $(p>0.07)$.

Norder Grusell et al. [40] evaluated the association between oral microbiome, EoE, and GERD. They cultivated both brush samplings and mucosal punch biopsies from the oral cavity and upper and lower esophagus from 17 subjects with GERD and 10 with EoE. The findings were compared to healthy control samples obtained from their previously published study using the same technique to survey the bacterial flora of the healthy human oral cavity and the upper and lower esophagus [41]. They found that alfaStreptococci (viridans Streptococcus including species such as; Streptococcus salivarius, Streptococcus mutans, Streptococcus mitis, Streptococcus sanguinis, and Streptococcus anginosus) was the most common group of bacteria in GERD, EoE, and healthy controls at all sample locations. No significant difference in species of the oral mucosa between EoE and healthy controls was seen. Overall, their results showed that GERD patients had less bacterial diversity in both oral and esophageal samples than EoE patients. Recently, Ziganshina et al. [42] used $16 \mathrm{~S}$ rRNA sequencing to investigate the complex nature of the salivary microbiota in patients with and without GERD. They noted that the relative abundances of Actinomyces (estimated as $3.5 \%$ vs. $2.5 \%)$, Atopobium (2.5\% vs. $1.5 \%)$, Stomatobaculum (0.7\% vs. $0.5 \%)$, Ruminococcaceae [G-2] (0.3\% vs. $0.1 \%)$, Veillonella (9.5\% vs. $7.5 \%)$, and Leptotrichia (5.5\% vs. $2.5 \%)$ were

Oral Microbiome and Esophageal

Diseases significantly higher in the saliva samples of patients with GERD, while the Porphyromonas (6.0\% vs. $8.5 \%$ ), Gemella (1.5\% vs. $2.0 \%)$, Peptostreptococcus ( $0.1 \%$ vs. $0.2 \%)$, and Neisseria ( $4.0 \%$ vs. $6.5 \%)$ were less abundant. There were no statistically significant differences in the diversity measured by Chaol, Shannon, and Simpson indices between the 2 study groups. Similarly, Wang et al. [43] assessed the differences in the salivary bacterial community composition between patients with GERD and healthy controls. Saliva samples from 55 patients with reflux esophagitis and 51 age- and sex-matched controls were analyzed via $16 S$ rDNA gene sequencing. The abundances of Prevotella, Veillonella, Megasphaera, Peptostreptococcus, Atopobium, Oribacterium, Eubacterium, and Lachnoanaerobaculum were increased, while Neisseria, Streptococcus, Rothia, Granulicatella, Gemella, Aggregatibacter, Treponema, Campylobacter, Filifactor, Corynebacterium, and Lactivibrio were decreased in the reflux esophagitis patients compared to the controls. This study did not find a significant change in the diversity of oral microbiota in reflux esophagitis patients (Simpson index, $p=0.60$; Shannon index, $p=0.38$ ).

\section{Esophageal Infections and Dysmotility}

While the association between oral candidiasis with candida esophagitis is a prime example of how oral microbiome dysbiosis can be intricately linked with an esophageal infection, we were unable to identify any publication examining this association. Similarly, no publications related to the association between the oral microbiome and infectious esophagitis (such as herpes simplex virus or cytomegalovirus infections) were identified. Furthermore, our search did not yield any publications describing the relationship between alterations in the oral microbiome and esophageal dysmotility (e.g., achalasia and megaesophagus).

\section{Discussion}

In this narrative review, we summarized the evidence from studies describing the association between alterations in the oral microbiota and esophageal conditions. Even though this area of investigation is in its nascency, there is emerging evidence to suggest that oral microbiome is altered in esophageal diseases. Deciphering the role of oral microbiome in pathogenesis, diagnosis, prognosis, and management of esophageal conditions can have significant clinical implications.

Oral microbiome analysis is an innovative and evolving field, particularly with the biotechnological advance- 
ments. Initial studies examining the oral microbiome were based on culture-dependent methods such as controlling laboratory conditions or culture medium, microscopy, carbohydrate fermentation tests, and antibiotic susceptibility [44]. These approaches relied primarily on the phenotypic biochemical characterization. They required a high skill level for optimal results and were time and resource intensive. Advances in the genomic techniques in 1980s enabled culture-independent study of diverse microbial communities [45]. This led to a fundamental change in approaching and understanding the human microbiome including the oral microbiome [46-48]. The culture-independent approach has given rise to many exciting fields such as metagenomics, metatranscriptomics, metaproteomics, and single-cell genomics whose applications have provided abundant information on the functional dynamics of microbial environments.

Most of the studies summarized in this narrative review used culture independent methods, specifically $16 \mathrm{~S}$ rRNA gene sequencing, to interrogate bacterial relative abundance and diversity in the clinical samples. The results suggest that the oral microbiome is unique for patients with esophageal malignancies, premalignancies, and benign conditions. Interestingly, the oral microbiome of patients with $\mathrm{BE}$ is dominated by gram-negative bacteria such as Fusobacterium, Prevotella, Dialister, Firmicutes, Lautropia, and Bacteroidales. As BE is one of the few known risk factors for esophageal cancer, the crosstalk between the oral microbiome and mechanism of pathogenesis of $\mathrm{BE}$ and subsequent EAC remains an exciting area for translational research. Likewise, discovering the interplay between the oral microbiome and mechanisms underlying the development of other esophageal conditions also remains promising. It is conceivable that discerning the importance of these associations can help in developing a framework for cancer likelihood or diagnosis based on the microbial microenvironment.

Understanding the cause and effect relationship and how microbes are mechanistically linked to the pathogenesis of the several esophageal diseases is an area of active investigation. The previously reviewed studies proposed several mechanisms thought to encourage esophageal conditions. One proposed mechanism is direct adhesion and subsequent invasion by the bacterium, which ultimately induces inflammation and alters the host immunity thereby encouraging the transition to dysplasia and ultimately promoting carcinogenesis [26, 27]. Additional possibilities include induction of a pro-inflammatory group of bacteria after loss of certain microbiomes leading to proliferation of other bacteria capable of opportunistic pathogenicity (such as Clostridium in the case of the colon) [35], or as a result of a direct interaction with the nutrients and esophageal binding sites [49]. Others suggest that diet plays a role and specifically food allergen-mediated eosinophilia may drive inflammation in the esophagus and may be linked to esophageal disease $[29,30,37,39]$. Though the current studies show associations between various microbes and esophageal diseases, no distinct pathogenetic mechanisms have been identified for these microbiome alterations.

Despite the promise, it is important to note that a direct comparison and analysis of the results among these studies can be challenging because of the variability in sample collection (mouthwash samples vs. saliva), the cross-sectional nature of the studies, and heterogeneity in their study design. Much of the research to date has focused on perturbations of the oral microbiome in esophageal cancerous, precancerous, and benign diseases. There is paucity of data related to oral microbiome and infectious esophageal conditions and esophageal motility disorders. Furthermore, there are epidemiological data to suggest that environmental factors and local factors can alter the oral microbiome. While most of the studies excluded individuals with periodontal/gingival diseases, there was no clear indication of how they addressed the confounding effects of many of the other variables.

While the $16 \mathrm{~S}$ rRNA sequencing remains the standard for bacterial taxonomic profiling at this time, it falls short in its ability to characterize viruses and fungi, bacteria at the species level, and it does not directly evaluate function or the interplay between the host and the microbe. Metagenomics and metatranscriptomics hold potential to give information not only about the microbial composition at the species level but it can also provide insight into involved metabolic pathways [50]. This can be valuable in assessing the causality, promoting novel diagnostic approaches, and developing a personal and precise approach, wherein clinicians can alter oral microbiome using drug development and targeted therapy to improve clinical outcomes. Furthermore, additional studies are needed to determine whether alterations in the microbiome can impact the natural history of various esophageal diseases.

\section{Conclusion}

We reviewed the current evidence available for alterations in oral microbiome in esophageal diseases. Emerging data suggest that there are distinct oral microbiome 
patterns associated with malignant, premalignant, and benign esophageal conditions. Further studies are needed to determine the causal relationship between oral microbiome and malignant, premalignant, and benign esophageal diseases. There is an opportunity for further research to discern the oral microbiome composition in esophageal infections and esophageal dysmotility conditions.

\section{Conflict of Interest Statement}

The authors have no conflicts of interest to declare.

\section{Funding Sources}

R.B. is supported by T32 training grant (5T32HD060554-10), the Thrasher Research Fund, and the Vanderbilt Institute for Clinical and Translational Research. S.R.D. is currently supported by NIAID (R21AI154016-01， R21AI149262， R21AI14232， R21AI14232101A1S1,U19AI095227, andP30AI110527),NHLBI(1R01HL146401), and start-up funds from Vanderbilt University Medical Center. G.H. is supported by the American College of Gastroenterology Junior Faculty Development Award and the Eunice Kennedy Shriver National Institute of Child Health \& Human Development of the National Institutes of Health under the award number: K12HD087023.

\section{Author Contributions}

Rachel Bernard contributed toward literature search, identifying relevant publications, data extraction, interpreting the results, drafting the manuscript, and designing the tables and figures. Irtiqa Fazili contributed toward literature search, identifying relevant publications, data extraction, interpreting the results, drafting the manuscript, and designing the tables. Seesandra V. Rajagopala contributed toward conceiving and designing the study, identifying relevant publications, data extraction, interpreting the results, and made critical contributions in the manuscript. Suman R. Das contributed toward conceiving and designing the study, data extraction, interpreting the results, and made critical contributions in the manuscript. Girish Hiremath contributed toward conceiving and designing the study, literature search, identifying relevant publications, data extraction, interpreting the results, drafting the manuscript, and designing the tables and figures. All authors have given final approval of the version to be published.

\section{References}

1 American Cancer Society. Cancer facts \& figures 2018. Am Cancer Soc. 2018.

2 Dellon ES, Jensen ET, Martin CF, Shaheen NJ, Kappelman MD. Prevalence of eosinophilic esophagitis in the United States. Clin Gastroenterol Hepatol. 2014 Apr;12(4):589-96.e1.

3 El-Serag HB, Sweet S, Winchester CC, Dent J. Update on the epidemiology of gastro-oesophageal reflux disease: a systematic review. Gut. 2014 Jun;63(6):871-80.

4 Hiergeist A, Gläsner J, Reischl U, Gessner A. Analyses of intestinal microbiota: culture versus sequencing. ILAR J. 2015;56(2):228-40.

5 Atarashi K, Suda W, Luo C, Kawaguchi T, Motoo I, Narushima S, et al. Ectopic colonization of oral bacteria in the intestine drives TH1 cell induction and inflammation. Science. 2017;358(6361):359-65.

6 Farrell JJ, Zhang L, Zhou H, Chia D, Elashoff D, Akin D, et al. Variations of oral microbiota are associated with pancreatic diseases including pancreatic cancer. Gut. 2012 Apr; 61(4):582-8.

7 Lv J, Guo L, Liu JJ, Zhao HP, Zhang J, Wang $\mathrm{JH}$. Alteration of the esophageal microbiota in Barrett's esophagus and esophageal adenocarcinoma. World J Gastroenterol. 2019 May 14;25(18):2149-61.

8 Corning B, Copland AP, Frye JW. The microbiome in health and disease. Curr Gastroenterol Rep. 2018 Aug 1;20(8):39.

9 Snider EJ, Freedberg DE, Abrams JA. Potential role of the microbiome in Barrett's esophagus and esophageal adenocarcinoma. Dig Dis Sci. 2016 Aug;61(8):2217-25.
10 Mannell A, Plant M, Frolich J. The microflora of the oesophagus. Ann R Coll Surg Engl. 1983 May;65(3):152-4.

11 Pei Z, Bini EJ, Yang L, Zhou M, Francois F, Blaser MJ. Bacterial biota in the human distal esophagus. Proc Natl Acad Sci U S A. 2004 Mar 23;101(12):4250-5.

12 Sampaio-Maia B, Caldas IM, Pereira ML, Pérez-Mongiovi D, Araujo R. The oral microbiome in health and its implication in oral and systemic diseases. Adv Appl Microbiol. 2016; 97:171-210.

13 Lim Y, Totsika M, Morrison M, Punyadeera C. Oral microbiome: a new biomarker reservoir for oral and oropharyngeal cancers. Theranostics. 2017 Sep 26;7(17):4313-21.

14 Ahn J, Chen CY, Hayes RB. Oral microbiome and oral and gastrointestinal cancer risk. Cancer Causes Control. 2012 Mar;23(3):399-404.

15 Dewhirst FE, Chen T, Izard J, Paster BJ, Tanner ACR, Yu W-H, et al. The human oral microbiome. J Bacteriol. 2010 Oct; 192(19): 5002-17.

16 Deo PN, Deshmukh R. Oral microbiome: unveiling the fundamentals. J Oral Maxillofac Pathol. 2019 Jan-Apr;23(1):122-8.

17 Gomez A, Espinoza JL, Harkins DM, Leong P, Saffery R, Bockmann M, et al. Host genetic control of the oral microbiome in health and disease. Cell Host Microbe. 2017;22(3):269.

18 Li J, Quinque D, Horz HP, Li M, Rzhetskaya M, Raff JA, et al. Comparative analysis of the human saliva microbiome from different climate zones: Alaska, Germany, and Africa. BMC Microbiol. 2014;14(1):316.
19 Percival RS, Challacombe SJ, Marsh PD. Agerelated microbiological changes in the salivary and plaque microflora of healthy adults. J Med Microbiol. 1991;35(1):5.

20 Takeshita T, Kageyama S, Furuta M, Tsuboi H, Takeuchi K, Shibata Y, et al. Bacterial diversity in saliva and oral health-related conditions: the Hisayama Study. Sci Rep. 2016;6: 22164.

21 Wu J, Peters BA, Dominianni C, Zhang Y, Pei $Z$, Yang L, et al. Cigarette smoking and the oral microbiome in a Large Study of American Adults. ISME J. 2016;10(10):2435.

22 Fan X, Peters BA, Jacobs EJ, Gapstur SM, Purdue MP, Freedman ND, et al. Drinking alcohol is associated with variation in the human oral microbiome in a Large Study of American Adults. Microbiome. 2018;6(1): 59.

23 Mishiro T, Oka K, Kuroki Y, Takahashi M, Tatsumi K, Saitoh T, et al. Oral microbiome alterations of healthy volunteers with proton pump inhibitor. J Gastroenterol Hepatol. 2018;33(5):1059.

24 Munn Z, Stern C, Aromataris E, Lockwood C, Jordan Z. What kind of systematic review should i conduct? A proposed typology and guidance for systematic reviewers in the medical and health sciences. BMC Med Res Methodol. 2018 Jan 10;18(1):5.

25 Moher D, Liberati A, Tetzlaff J, Altman DG; PRISMA Group. Preferred reporting items for systematic reviews and meta-analyses: The PRISMA statement. PLoS Med. 2009 Jul 21;6(7):e1000097. 
26 Narikiyo M, Tanabe C, Yamada Y, Igaki H, Tachimori $Y$, Kato $H$, et al. Frequent and preferential infection of Treponema denticola, Streptococcus mitis, and Streptococcus anginosus in esophageal cancers. Cancer Sci. 2004 Jul;95(7):569-74.

27 Kageyama S, Takeshita T, Takeuchi K, Asakawa M, Matsumi R, Furuta M, et al. Characteristics of the salivary microbiota in patients with various digestive tract cancers. Front Microbiol. 2019 Aug 2;10:1780.

28 Zhao Q, Yang T, Yan Y, Zhang Y, Li Z, Wang $\mathrm{Y}$, et al. Alterations of oral microbiota in chinese patients with esophageal cancer. Front Cell Infect Microbiol. 2020;10:541144.

29 Chen X, Winckler B, Lu M, Chen H, Yuan Z, Yang Y, et al. Oral microbiota and risk for esophageal squamous cell carcinoma in a high-risk area of China. PLoS One. 2015 Dec 7;10(12):e0143603.

30 Wang Q, Rao Y, Guo X, Liu N, Liu S, Wen P, et al. Oral microbiome in patients with oesophageal squamous cell carcinoma. Sci Rep. 2019 Dec 13;9(1):19055.

31 Chen MF, Lu MS, Hsieh CC, Chen WC. Porphyromonas gingivalis promotes tumor progression in esophageal squamous cell carcinoma. Cell Oncol. 2020 Apr;44(2):373-84.

32 Meng F, Li R, Ma L, Liu L, Lai X, Yang D, et al. Porphyromonas gingivalis promotes the motility of esophageal squamous cell carcinoma by activating NF- $\kappa \mathrm{B}$ signaling pathway. Microbes Infect. 2019;21(7):296.

33 Peters BA, Wu J, Pei Z, Yang L, Purdue MP, Freedman ND, et al. Oral microbiome composition reflects prospective risk for esophageal cancers. Cancer Res. 2017 Dec 1;77(23): 6777-87.
34 Kawasaki M, Ikeda Y, Ikeda E, Takahashi M, Tanaka D, Nakajima Y, et al. Oral infectious bacteria in dental plaque and saliva as risk factors in patients with esophageal cancer. Cancer. 2021;127(4):512.

35 Snider EJ, Compres G, Freedberg DE, Giddins MJ, Khiabanian $\mathrm{H}$, Lightdale CJ, et al. Barrett's esophagus is associated with a distinct oral microbiome. Clin Transl Gastroenterol. 2018 Feb 20;9(3):135.

36 Okereke IC, Miller AL, Hamilton CF, Booth AL, Reep GL, Andersen CL, et al. Microbiota of the oropharynx and endoscope compared to the esophagus. Sci Rep. 2019 Jul 15;9(1): 10201.

37 Benitez AJ, Hoffmann C, Muir AB, Dods KK, Spergel JM, Bushman FD, et al. Inflammation-associated microbiota in pediatric eosinophilic esophagitis. Microbiome. 2015 Jun 1; $3: 23$.

38 Liacouras CA, Furuta GT, Hirano I, Atkins D, Attwood SE, Bonis PA, et al. Eosinophilic esophagitis: updated consensus recommendations for children and adults. J Allergy Clin Immunol. 2011 Jul;128(1):3-20.e6; quiz 21-2.

39 Hiremath G, Shilts MH, Boone HH, Correa $\mathrm{H}$, Acra S, Tovchigrechko A, et al. The salivary microbiome is altered in children with eosinophilic esophagitis and correlates with disease activity. Clin Transl Gastroenterol. 2019Jun;10(6):e00039.

40 Norder Grusell E, Dahlén G, Ruth M, Bergquist $\mathrm{H}$, Bove $\mathrm{M}$. The cultivable bacterial flora of the esophagus in subjects with esophagitis. Scand J Gastroenterol. 2018 Jun;53(6): 650-6.

41 Norder Grusell E, Dahlén G, Ruth M, Ny L, Quiding-Järbrink M, Bergquist $\mathrm{H}$, et al. Bacterial flora of the human oral cavity, and the upper and lower esophagus. Dis Esophagus. 2013 Jan;26(1):84-90.
42 Ziganshina EE, Sagitov II, Akhmetova RF, Saleeva GT, Kiassov AP, Gogoleva NE, et al. Comparison of the microbiota and inorganic anion content in the saliva of patients with gastroesophageal reflux disease and gastroesophageal reflux disease-free individuals. Biomed Res Int. 2020 May 13;2020:2681791.

43 Wang B, Zhang Y, Zhao Q, Yan Y, Yang T, Xia $Y$, et al. Patients with reflux esophagitis possess a possible different oral microbiota compared with healthy controls. Front Pharmacol. 2020;11:1000.

44 Krishnan K, Chen T, Paster BJ. A practical guide to the oral microbiome and its relation to health and disease. Oral Dis. 2017 Apr; 23(3):276-86.

45 Handelsman J. Metagenomics: application of genomics to uncultured microorganisms. Microbiol Mol Biol Rev. 2004 Dec;68(4):669-85.

46 Rappé MS, Giovannoni SJ. The uncultured microbial majority. Annu Rev Microbiol. 2003;57:369-94.

47 Altmäe S, Franasiak JM, Mändar R. The seminal microbiome in health and disease. Nat Rev Urol. 2019 Dec;16(12):703-21.

48 Methé BA, Nelson KE, Pop M, Creasy HH, Giglio MG, Huttenhower C, et al. A framework for human microbiome research. $\mathrm{Na}$ ture. 2012 Jun 13;486(7402):215-21.

49 Kelly D, Yang L, Pei Z. A review of the oesophageal microbiome in health and disease. Methods in microbiology; 2017.

50 Baker JL, Bor B, Agnello M, Shi W, He X. Ecology of the oral microbiome: beyond bacteria. Trends Microbiol. 2017 May;25(5):36274. 